\title{
Perspectiva integral sobre el desarrollo educativo costarricense
}

\section{Integral Perspective of the Costa Rican Educational Development}

\author{
Maureen Camacho Oviedo \\ División de Educación Básica del Centro de Investigación y Docencia en Educación \\ Universidad Nacional \\ Heredia, Costa Rica
}

Recibido 22 de marzo de 2010 • Aceptado 24 de junio de 2010

\begin{abstract}
Resumen. El propósito de este ensayo es plantear el desarrollo educativo que se ha experimentado en nuestro país, esbozando aspectos influyentes en él, desde los planos político, social, religioso y económico. Pretende informar sobre datos estadísticos como indicadores del desarrollo de nuestro sistema educativo, en aspectos de cobertura, escolaridad promedio, repitencia, deserción, entre otros. Es imperante lograr cambios o reestructuraciones en nuestro sistema educativo en todos los niveles, desde el preescolar hasta el universitario, por lo que debe tomarse en consideración el aporte de todos los involucrados en el sector educativo, pues el recurso humano es valioso y deben escucharse todas sus propuestas innovadoras, en especial aquellas que pretenden que construyamos juntos y no las ideas impuestas que obstaculicen el desarrollo educativo de nuestro país. Las mejoras deben darse, no sólo porque son necesarias, sino porque existe en muchos el deseo de optimizar y de alcanzar un auténtico enriquecimiento de nuestra educación.
\end{abstract}

Palabras clave. Desarrollo educativo, educación, reforma educativa, cobertura, obligatoriedad, repitencia, deserción, innovación.

Abstract. The purpose of this essay is to raise the educational development that has been experienced in our country, outlining influential frames such as political, social, religious and economical ones. It aims to report statistical data as indicators of the development of our educational system, in areas of coverage, average education, repetition, desertion, among others. It is imperative to achieve changes and restructuring in our education system at all levels, from kindergarten to university, so it should take into account the contribution of all stakeholders in the education sector, as the human resource is valuable and all the innovative proposals should be heard, especially those that seek to build together and not ideas imposed that hinder the development of education in our country. Improvements must be necessary because there is an existing desire by many people of improving and achieving a true enrichment in our education.

Key words. Educational development, education, educational amend, coverage, binding, repetition, desertion, innovation.

\footnotetext{
Máster en Educación con Énfasis en Docencia Universitaria, otorgado por la Universidad Nacional, Costa Rica. Licenciada en Ciencias de la Educación con concentración en Educación Básica I y II Ciclos. Ha laborado como docente de primaria en centros educativos públicos y privados. Actualmente, se desempeña como Académica de la División de Educación Básica (DEB) del Centro de Investigación y Docencia en Educación (CIDE) de la Universidad Nacional (UNA), Costa Rica, donde ha desempañado diversas funciones: en el área de docencia, en cursos de los diferentes niveles de la Carrera de Pedagogía con énfasis en I y II Ciclos de la Educación General Básica y a nivel de diplomado de la Carrera de Educación Especial con énfasis en Integración; en la Coordinación de la Intervención Pedagógica a nivel de Diplomado de la Carrera de Pedagogía con énfasis en I y II Ciclos; en los procesos de supervisión de la práctica a nivel de Bachillerato de la Carrera de Pedagogía con énfasis en I y II Ciclos. Ha sido miembra del Proyecto de Desarrollo Académico de la División de Educación Básica (DEB) del Centro de Investigación y Docencia en Educación (CIDE) de la Universidad Nacional (UNA), Costa Rica, y responsable de la Carrera de Pedagogía con énfasis en I y II Ciclos.Correo electrónico: mcamac@una.ac.cr
} 
El desarrollo educativo puede ser visualizado desde diversas perspectivas. Una valiosa concepción de este consiste en definirlo como un proceso sistemático, que erigirá al ser humano como resultado de la síntesis de la interacción de diferentes elementos y del dinamismo de diversas fuerzas sociales. El desarrollo educativo busca ampliar las oportunidades de progreso y de realización de los seres humanos, al fomentar la potenciación de sus capacidades y habilidades cognitivas, físicas, sociales, emocionales y culturales, adaptadas al cambio constante de la realidad educativa.

El desarrollo educativo debe ser un proceso que permita la transformación y mejoría de calidad de la educación. Para ello, este proceso sintetiza tres concepciones de la educación, a saber:

1. El derecho de las personas a poder educarse; a explorar sus capacidades; a identificar sus necesidades; a generar conocimientos, y a contar con los elementos y las condiciones necesarias para lograrlo, las cuales implican cobertura, infraestructura, materiales didácticos, profesionales en educación, igualdad de oportunidades y de acceso.

2. La educación como eje de las políticas de desarrollo e inversión de un país, como reconocimiento del Estado al derecho a la educación.

3. La educación como elemento transmisor de la idiosincrasia de un país y de la cultura mundial, que permita a los individuos y grupos mejorar, al concientizar sobre su responsabilidad social de educarse y educar, ya que a todos les corresponde la obligación de construir y de buscar el desarrollo educativo y promover el de quienes les rodean, esto es: de quienes conforman el país y el planeta.

En esta conceptualización se debe tener en cuenta que el desarrollo educativo no es estático, sino un proceso cambiante, el cual ha ido dinamizándose con el tiempo. Lo anterior se justifica gracias a que nuestra educación se ha visto y se verá influida en su desarrollo, por tres grandes fuerzas que han intervenido en ella a través de los años, en ocasiones para hacerla avanzar y en otros momentos para limitarla. Estas influencias son: la religión católica, la política filosófica en el ámbito mundial y la economía desplegada en el país. Nuestro desarrollo educativo es la síntesis del aporte cooperativo de esas tres fuerzas, las cuales continuarán constituyendo la educación, que siempre ha pretendido para Costa Rica una senda de mayor progreso.

El sistema educativo, como elemento estatal para proveer educación escolarizada a las personas, es uno de los mecanismos en que se ven inmersos, de una manera u otra, en una proporción u otra, todos los habitantes de un país. Por esto, todo aquello que influya en el desarrollo del Estado, tanto en el nivel nacional como en el internacional, va a repercutir en la educación que este brinda.

Las luchas ideológicas más reconocidas en el mundo, tales como la Revolución Industrial, la Revolución Francesa, la Independencia de los Estados Unidos, han afectado el proceso histórico de nuestro país y, por ende, el de nuestra educación, la cual se ha caracterizado, a través del tiempo, según los momentos históricos independentistas vivenciados. Así pues, la educación es permeada por las situaciones sociales, culturales, económicas y políticas de un país.

En 1821, se logra la independencia de las colonias españolas, nuestro país deja de ser provincia y se ve en una encrucijada, pues durante el período de la Colonia, Costa Rica había sido una de las provincias más pobres y atrasadas, dependientes de las decisiones españolas, incluyendo las decisiones en lo educativo. Al lograr la independencia, sobresale la necesidad de tomar, como país, decisiones propias que permitieran construir una identidad nacional y una estructura productiva capaz de sacarlo adelante. Pero como, en ese momento, no se poseían los recursos humanos ni materiales necesarios para ello, se consideró a la educación como el principal instrumento social que permitiría formar ciudadanos con las aptitudes y las características necesarias para producir un cambio positivo hacia el desarrollo. 
A partir de la independencia, el país debió organizar su sistema de gobierno democrático; pero, para ello, necesitaba ciudadanos preparados, los que, al menos, pudiesen leer y escribir, pues sin habitantes educados no se podía construir una república. Hubo una conciencia de la relación directa entre la educación y el progreso del país. Incluso la Junta Superior Gubernativa, en 1824, declaró la Instrucción Pública como la base y principal fundamento de la felicidad humana y prosperidad común (Dengo, 1995).

Una de las prioridades era conseguir que el país saliera del atraso en el que se encontraba; para ello, se llevaron a cabo varias acciones. Entre estas se pueden mencionar las siguientes:

a. Un aporte económico por parte del Gobierno para la Casa de Enseñanza de Santo Tomás, para que reorganizara su plan de estudios y se convirtiera en el primer centro de estudios superiores del país.

b. La organización de la enseñanza en un sistema descentralizado a cargo de los gobiernos locales.

c. La introducción, en 1830, de la imprenta.

d. Una mayor concientización sobre la obligatoriedad de la enseñanza.

e. La creación de más instituciones educativas, conforme el aumento de la capacidad financiera del Estado, producto del crecimiento de la actividad cafetalera.

Estas acciones permitieron ir superando, al menos inicialmente, la incertidumbre surgida luego de la independencia. Además, a partir de 1840, se originan otros hechos en relación con el desarrollo educativo costarricense, uno importante fue la transformación de la Casa de Enseñanza de Santo Tomás, en 1844, en la Universidad de Santo Tomás. Según Dengo (1995), "a partir de entonces tuvo un relevante papel cultural y su acción formativa de los principales hombres del siglo XIX hizo, inclusive, que los costarricenses que aspiraban a una educación superior no tuvieran que salir a procurársela en otros países centroamericanos, como anteriormente había sido" (p. 89).

Por su origen y su función cultural, la creación de esta Universidad fue un hecho realmente significativo dentro de los logros del doctor José María Castro Madriz en el campo educativo, el cual era, en ese momento, Ministro General de la administración de José María Alfaro. En 1849, como Presidente de la República, promulgó un Reglamento Orgánico del Consejo de Instrucción Pública, el cual permitió, entre otros avances, la difusión de la educación primaria y media de las mujeres costarricenses.

El desarrollo en el ámbito educativo continuó, pues durante el período de 1890 a 1948, el país se transformó, rápidamente, en los aspectos económico, cultural y social, gracias al impulso que tuvo el comercio exterior con la exportación de café, de banano y de cacao. En el campo educativo, además, el presidente de Costa Rica, doctor Jesús Jiménez, ya había logrado que se declarara en la Constitución Política de 1869, la enseñanza primaria para ambos sexos obligatoria, gratuita y costeada por la Nación.

En 1884, con la aprobación de las Leyes liberales, se establece la separación de los campos de acción de la Iglesia Católica y del Estado. Entre estas leyes liberales, se incluye, según Gamboa y Vásquez (1998), la prohibición a los sacerdotes de hacerse cargo de la dirección de escuelas y de colegios del Estado. La promulgación de la enseñanza laica es otro aspecto importante en el desarrollo educativo, pues desde tiempos de la colonia imperaba la influencia de la Iglesia en asuntos educativos. Este sistema de enseñanza primaria centralizado, secular, gratuito y obligatorio, pretendía "instruir a los hijos de artesanos, trabajadores y campesinos en nuevos valores y destrezas" (Molina y Palmer, 2007, p. 70). Sin embargo, debe considerarse que si bien se suprimió la influencia 
de la Iglesia en la educación, no se desvirtúa el aporte que esta puede haber dado a la formación de las personas. Esta supresión de la enseñanza religiosa impactó la matrícula de la educación primaria y varió hasta 1890, cuando se reinstituye la enseñanza religiosa en las escuelas públicas del país. Además, en la actualidad, se sigue tomando en cuenta y se imparte en las escuelas públicas lecciones de Educación religiosa, y en muchos centros privados se da un impulso a la formación espiritual o axiológica, aunque no se circunscriben, totalmente, a la enseñanza religiosa católica, pues se ofrece un espacio más flexible para el fomento de valores, sin limitarse a una religión en particular.

En 1886, durante la administración de Bernardo Soto, el secretario de Instrucción Pública y Hacienda de su gobierno, el licenciado Mauro Fernández, realizó una importante reforma educativa mediante la Ley General de Educación Común, con la que logra la reestructuración de la educación de la época y, por tanto, convierte este momento en uno de los procesos característicos de nuestra historia educativa y política; pues como señala Salazar (2003): “...los cambios verificados en el ámbito de la enseñanza nacional a partir de 1885, respondieron de manera específica a los requerimientos del proyecto político de corte liberal que sustentaba el grupo de poder" (p. 82).

Unas de las medidas que se tomaron con esta reforma fue el cierre de la Universidad de Santo Tomás y la creación, en su lugar, de una Escuela Profesional de Derecho, pues la Universidad fue clausurada, pero continuó con parte de sus funciones profesionales y docentes, al mantener la Facultad de Derecho. Fernández justificó estas medidas, en el hecho de que antes de fortalecer la educación superior, se debía reorganizar la educación primaria y la secundaria. Con esta clausura, no se pretendía mutilar la educación superior, sino "...establecer una universidad... más comprometida con la resolución de los problemas más apremiantes de la sociedad costarricense" (Salazar, 2003, p. 83). Además, el señor Mauro Fernández pretendía darle un verdadero impulso a la educación primaria costarricense, con la mayor cobertura posible, lo que permitiría satisfacer el mandato constitucional y, a la vez, reforzar la educación secundaria, al enviar estudiantes mejor preparados a los colegios. En relación con esto, en el nivel de secundaria se fundan importantes instituciones: el Instituto de Alajuela y el Liceo de Costa Rica, con “...la Escuela Normal para varones, adscrita como una sección" (Dengo, 1995, p. 101), en 1887, y el Colegio Superior de Señoritas, en el año 1888, en el cual se estableció una Escuela Normal para mujeres.

Ante la necesidad de seguir formando docentes capacitados, se crea en 1915 la Escuela Normal, la cual fue cuna de grandes educadores; con ella, se pretendía lograr cambios en el plano de la educación, y se conceptualiza desde un punto de vista más social y político, lo que se ajustaba al modelo agro exportador de la época. Posteriormente, acontecen otros importantes hechos en el nivel de la educación superior: la apertura de la Universidad de Costa Rica, en 1941 y de la Universidad Nacional en 1973, con lo que se abren relevantes oportunidades, en diferentes momentos, para los habitantes de nuestro país.

Entre 1940 y 1980, la educación técnica, también, recibió un gran impulso, pues se valora su importancia y sus ventajas, para las personas que deseaban acceder a este tipo de educación. Se pretende formar individuos con una especialidad técnica vocacional, que posean, como señala Salazar (2003), perfiles que respondan a las demandas del sistema, pues a pesar de los esfuerzos que hiciera el Ministerio de Educación Pública, estos resultaron insuficientes, por lo que "...se recurre a la formación de instituciones de capacitación o se realizan convenios de apoyo mutuo con instancias privadas o estatales, nacionales o extranjeras" (Salazar, 2003, pp. 341-342). Incluso, dentro de esta área de educación técnica, se crea el 21 de mayo de 1965, el Instituto Nacional de Aprendizaje, con el fin de brindar a sus alumnos capacitación y especialización para realizar trabajos manuales y técnicos.

También, cabe señalar que en el desarrollo educativo de nuestro país, han influido diversas corrientes y movimientos pedagógicos provenientes del extranjero, a saber: la misión pedagógica 
chilena, el movimiento de Escuela Nueva, la visita de pensadores guatemaltecos y cubanos, la influencia del estilo educativo europeo y estadounidense, entre otros.

Actualmente, nuestra educación se rige por una estructura ya establecida, en cuanto a la jerarquía y los órganos rectores de todo el sistema educativo; posee un sistema organizado de escogencia de personal docente, un programa de estudios estandarizado, una serie de convenios internacionales, leyes, reglamentos y normativas, y con una calidad en infraestructura, mobiliario y material didáctico disponible, aspectos que se pueden mejorar.

Nuestro acontecer histórico educativo ha sido moldeado por diferentes influencias ideológicas, políticas, económicas, religiosas, que pueden ser ubicadas en el tiempo y en el espacio. Estas han generado cambios y eventos relevantes, que permiten comprender el porqué de nuestro desarrollo educativo, el cual ha sido impactado por pensamientos de personalidades que figuraron en los planos político y social. Personalidades que corresponden a seres humanos de alto conocimiento y renovadoras ideas, influidas por corrientes de pensamiento más liberales, positivistas, racionalistas, cientificistas, con propuestas de cambio. Tras un diagnóstico de la situación educacional del país, en sus diversos ámbitos, deciden hacer reformas o implementar planes aprendidos u observados en otros países con mayor desarrollo educativo, teniendo en cuenta las realidades y las necesidades imperantes en el país, con el fin de generar progreso en la educación nacional y, por tanto, en la sociedad en general.

Otro aspecto que nos permite ubicar la situación de nuestro desarrollo educativo es el estudio y análisis de datos estadísticos, que reflejan, numéricamente, la realidad educativa reciente y actual. Es notorio que en el sistema educativo se hayan dado importantes esfuerzos por lograr su mejoría. Sin embargo, también persisten atrasos considerables que han afectado el desarrollo de las personas $\mathrm{y}$, por ende, del país en general. Lo anterior se puede analizar mediante el estudio de datos como la cobertura, la obligatoriedad, las diferencias entre los sectores público y privado, los porcentajes de repitencia y de deserción.

Estos datos permiten comprender, desde otro punto de vista, el desarrollo o la paralización que podría estarse evidenciando en nuestro sistema educativo, ya que permiten detectar cuáles renovaciones necesita la educación costarricense, al cifrar situaciones propias del quehacer educativo.

Para lograr que la población alcance, como mínimo, la educación general básica y efectuar lo estipulado en el artículo 78 de nuestra Constitución Política ${ }^{2}$, el Estado ha buscado un avance en la cobertura del sistema de educación formal. Sin embargo, no se cumple, en su totalidad, con la expectativa de obligatoriedad de este artículo y el nivel de cobertura disminuye conforme se avanza en los ciclos educativos. Para el año 2006, según el Programa Estado de la Nación en Desarrollo Humano Sostenible (2007), los indicadores de cobertura, rendimiento, repitencia y brechas del sistema educativo formal no mostraron cambios relevantes, con respecto a las principales tendencias reseñadas en los últimos informes.

La cobertura en primaria es alta, aunque no equitativa para I y II Ciclos, los que, respectivamente, señalan un 100,3\% y un 88,8\% para el 2002 (Programa Estado de la Nación en Desarrollo Humano Sostenible, 2003), lo cual se ha establecido como uno de los primeros indicadores del incumplimiento de la obligatoriedad de la educación general básica.

Esta diferencia es aún más marcada al pasar a la secundaria, con una cobertura de $64,9 \%$ para III Ciclo y un 36\% para la educación diversificada, en ese mismo año. No obstante, como menciona el Programa Estado de la Nación en Desarrollo Humano Sostenible (2009), se debe poner más

En el Art. 78 de la Constitución Política se señala que la educación preescolar, general básica y diversificada son obligatorias, y en el sistema público, gratuitas y costeadas por el Nación, según reforma de la Asamblea Legislativa de mayo de 2010. 
empeño en incrementar la cobertura del cuarto ciclo, para poder, así, asegurar el éxito académico de los estudiantes, pues esta sigue siendo baja e insuficiente, al pasar de un 36\% en el 2002 a un $44,2 \%$ en el 2008.

Sin duda, existe un gran inconveniente en la educación secundaria, pues no se logra mantener a los jóvenes dentro del sistema y los que sí permanecen sufren situaciones de repitencia, o se trasladan a sistemas de educación abiertos, nocturnos o no formales, los cuales, según su consideración, pueden permitirles obtener su título de educación media.

El Programa Estado de la Nación en Desarrollo Humano Sostenible (2003) menciona que, según el Ministerio de Educación Pública (MEP) y las estimaciones del Censo de Población del 2000, resulta palpable un logro significativo en la educación preescolar, tanto en su nivel interactivo como en el de transición. La cobertura, para el 2002, en preescolar, alcanzó un 86,9\% y ha ido en aumento desde ese momento, lo que indica que los padres y las madres de familia comprenden la importancia de iniciar la educación temprana en los niños, pues son conscientes de que la mayor riqueza se encuentra en la primera infancia, y que esta es la base para el posterior desarrollo educativo del individuo. Confían en la educación brindada para esta etapa por el sistema educativo.

De acuerdo con el Programa Estado de la Nación en Desarrollo Humano Sostenible (2008), el país presenta dificultades para lograr que los niños y las niñas culminen con éxito su paso por el sistema educativo. Entre el $85 \%$ y el $90 \%$ del estudiantado lograron concluir el nivel de primaria; sin embargo, sólo entre el 50\% y el 58\% de los alumnos que ingresó a primer grado pudieron terminar el nivel escolar sin repetir ningún año. En secundaria, la eficiencia es aún menor, pues solamente una cuarta parte de los estudiantes que inician en sétimo año consigue graduarse. Por ejemplo, el MEP estima que de cada mil niños de la cohorte del 2007, sólo 381 se graduarán.

Es necesario destacar que el nivel en el que se observa la mayor deserción sigue siendo en sétimo año: con un 19,5\%, en el año 2007 (Programa Estado de la Nación en Desarrollo Humano Sostenible, 2008); nivel que constituye el inicio de un nuevo ciclo. Lo anterior refleja la presencia de un abismo entre los niveles de primaria y de secundaria, pues no existe un adecuado proceso de transición, que tenga como objetivo primordial la adaptación del estudiante al nuevo ciclo de estudios. Esta situación, incluso, se presentaba cuando las pruebas nacionales de sexto grado, establecidas por el MEP, se aplicaban, las cuales constituían el perfil de salida académico de la primaria y certificaban que el alumno estaba preparado para ingresar al siguiente nivel. Aunque estas pruebas certificaran y permitieran el paso a la educación secundaria, no implicaban, precisamente, que los escolares tuvieran un manejo adecuado de todos los conocimientos básicos para enfrentarse al sétimo grado, pues los datos reflejan gran repitencia y deserción en este nivel. No se podría generalizar que todas las instituciones educativas escolares tuvieran las mismas posibilidades y características contextuales para preparar de igual manera a los estudiantes para las pruebas, y para enfrentar la secundaria con mayores posibilidades de éxito.

Además, los estudiantes, al pasar al colegio, se enfrentan a un estilo de estudio diferente al experimentado durante sus años de escuela, por lo que muchos no logran adaptarse, por completo, a las exigencias y complejidades de la secundaria. Entre los aspectos que influyen en esto se pueden mencionar algunos de tipo académico: mayor cantidad de asignaturas en el currículo, estilo de enseñanza diferente por parte de los profesores, entre otros; así como aspectos de índole, incluso, geográfico y económico.

En la secundaria, la mayoría de los jóvenes que desertan del sistema lo hacen a causa de factores económicos, familiares, sociales o factores propios del sistema educativo, los que, según el Departamento de Investigación Educativa del MEP, se relacionan con la inadecuada estructuración de los planes curriculares por grados o niveles, la desigual carga académica por materia, la falta 
de capacitación docente, la poca disponibilidad de materiales y recursos didácticos en el aula, la escasa asesoría a los estudiantes, la falta de talleres, laboratorios y bibliotecas, el ausentismo de los docentes y el desconocimiento de técnicas de estudio y metodologías de trabajo.

Se plantea en el Programa Estado de la Nación en Desarrollo Humano Sostenible (2007) que la deserción de los alumnos de secundaria, por razones vinculadas a problemas económicos (salen a trabajar) y de calidad de la enseñanza, aumentó de 12,5\%, en 2005, a 13,2\%, en el 2006. Esta tendencia, entre 2001 y 2003, se había logrado reducir, pero volvió a incrementarse a partir de 2004.

Además, los empleos en los que laboran estos jóvenes que desertan, muchas veces, son puestos laborales inestables con bajas remuneraciones, los cuales no les permiten una mejor calidad de vida. Generalmente, los padres de estos jóvenes no han tenido la oportunidad de una escolaridad completa y no promueven en sus hijos la relevancia del estudio, y la desestiman como un potencial mecanismo de movilidad social.

Para 2006, según el Programa Estado de la Nación en Desarrollo Humano Sostenible (2007), la escolaridad promedio de las personas de 18 a 64 años de edad fue de 8,6 años, lo que equivale a un aumento en la escolaridad, aunque sólo haya sido de 1,4 años en relación con 1990. Y para 2008, siguió aumentando, pues los años de escolaridad promedio de la población en edad activa se mantuvo en $8,8 \%$, lo que ubica a Costa Rica en un nivel intermedio en relación con otros países del mundo, aunque también revela que debe mejorarse este indicador (Programa Estado de la Nación en Desarrollo Humano Sostenible, 2009). Sin embargo, un hecho relevante es el adelanto que mostró el porcentaje de esta población que posee secundaria completa o más, al pasar de $25,1 \%$ en el año 1990 a $35,1 \%$ en el 2006.

Cabe indicar, además, que en los últimos diecisiete años disminuyó el porcentaje de jóvenes, entre 12 a 24 años, que no estudia ni trabaja, de 20,9\% en 1990 a 12,2\% en 2006. Lo anterior demuestra que los jóvenes, e incluso sus padres, han ido concientizándose de la importancia del estudio y de obtener su titulación básica y media, para poder obtener altas probabilidades de un empleo justo y mejor remunerado que les permita una superior calidad de vida. Además, refleja la importancia que representa para la juventud el tener una posibilidad de ingresos económicos, pues muchos jóvenes provienen de familias que no pueden cubrirles sus gastos básicos de alimentación, vestuario, estudio, tratamientos médicos, por lo que muchos optan por trabajar.

En la educación superior, aunque no existen datos de las universidades privadas, la cobertura ha aumentado, ya que desde 1990 se ha dado una gran expansión de estos establecimientos, que pasaron de ser ocho a más de 50 instituciones universitarias privadas; aunque su auge no ha ido acompañado de un marco de regulación en cuanto a la calidad académica.

Esta expansión de las universidades privadas se da en el marco de restricción de los establecimientos universitarios estatales, pues cada vez se exigen más pruebas y requisitos de admisión y los cupos son limitados, por lo que muchos jóvenes, en su afán de aprender y, sobre todo, de obtener un título universitario que les respalde para lograr un trabajo profesional, recurren a las carreras que ofrecen las universidades privadas, aunque, en muchos casos, su preparación académica sea diferente a la de las universidades públicas. Un mecanismo de apoyo con el que cuentan las universidades (tanto estatales como privadas) es el de realizar procesos de acreditación en sus diferentes carreras, proceso regido por el Sistema Nacional de Acreditación de la Educación Superior en Costa Rica (SINAES).

Podría señalarse que todos estos factores, relacionados con la deserción y la repitencia, son impedimentos para una mejor calidad y eficiencia educativa; pero no constituyen, por sí mismos, obstáculos insuperables para los jóvenes con espíritu y deseos de superación. Esto se refleja en todos 
aquellos estudiantes que se mantienen dentro del sistema y logran graduarse en los diferentes ciclos en los años establecidos, en aquellos que se mantienen a pesar de las dificultades y los niveles de deserción y de repitencia, o por el hecho de enfrentarse a las pruebas nacionales de Bachillerato, las que, muchas veces, además de sólo probar la capacidad memorística y aplicativa de un estudiante, es la forma de coartar las posibilidades de superación de las personas. Mientras el estudiante no las aprueba, ve limitado su acceso a la educación universitaria y a puestos laborales con mejor posición y reconocimiento económico, por lo que se requiere de una mayor constancia de los jóvenes por seguir esforzándose para concluir sus estudios secundarios y no desertar del sistema.

Un esfuerzo actual del MEP para minimizar el impacto de la deserción y la repitencia en los niveles de secundaria es la aplicación de la Reforma integral de las normas reguladoras de la promoción y repitencia dentro del sistema educativo público. Este documento, planteado por el Ministro de Educación actual, señor Leonardo Garnier Rímolo, fundamenta la reforma en las siguientes propuestas, aprobadas por el Consejo Superior de Educación:

a. Los estudiantes repetirán solamente las asignaturas que reprueban, y podrán seguir avanzando en asignaturas de niveles superiores.

b. Los cursos se aprobarán con la nota ponderada anual.

c. La evaluación de la conducta no afectará la evaluación académica, sino que se promoverá la corrección de la mala conducta y se combatirá sus causas.

d. Se desarrollarán medidas o esquemas de apoyo para los estudiantes repitentes y rezagados (Costa Rica, Ministerio de Educación Pública, 2009).

Para Garnier, esta reforma apunta a demostrar que la calidad no tiene por qué ser antítesis de la inclusión educativa, sino lo contrario, es la expulsión injustificada la que realmente atenta contra el derecho de todos a una educación de calidad (Costa Rica. Ministerio de Educación Pública, 2009). Esta reforma está siendo aplicada, en el nivel de secundaria, desde el 2009; pero habría que darle un período mayor de ejecución, así como de seguimiento y de sistematización de sus resultados, para justipreciar el impacto real que puede tener en el rendimiento final de los estudiantes y en la disminución de los índices de deserción y repitencia.

Costa Rica, con esfuerzo, compromiso y voluntad política, puede salir adelante. A pesar de las dificultades, en nuestro país existe el recurso humano apropiado para organizar y reestructurar el sistema educativo. Hay personas muy preparadas, conscientes de la realidad, objetivas en cuanto a las condiciones con las que se cuenta y las carencias que se pueden, factiblemente, satisfacer, para un verdadero relanzamiento de la educación costarricense, que responda, a la vez, a las exigencias de la sociedad actual tanto en el ámbito nacional como en el internacional.

Es posible que ciertos grupos económicos y políticos, como empresarios de alto nivel y grupos políticos establecidos en el país, desde hace muchos años, con importantes influencias en el campo financiero y gubernamental, no consideren necesario el mejoramiento de la educación, en especial la educación pública, ni tampoco la promoción de la igualdad de oportunidades de acceso a la educación para todos, ya que no les es funcional que todas las personas tengan la posibilidad de estudiar, y que transformen su visión de la vida, pues dejarían de constituir un grupo de mano de obra barata y fácil de manipular, según sus intereses. El mejorar el acceso y la calidad de la educación favorecería la movilidad social de amplios sectores de la sociedad, pero si fuera así, se pondría en cierta peligrosidad la estabilidad de esos grupos y tendrían más resistencia en la ejecución de propuestas, que más que favorecer a muchos, por lo general, favorecen a unos pocos. 
Cada costarricense debería tener la oportunidad de decidir lo que desea hacer en el campo académico y laboral, no debería verse limitado en sus deseos de continuar sus estudios. Si bien, no todos desean formalizar estudios universitarios, lo apropiado sería que esto se dé por la propia decisión de las personas y no porque no lograron obtener un cupo en una carrera. Todos los trabajos son dignos e, igualmente, valiosos para la humanidad; pero alguien que labore en un campo que no desee no será una persona feliz, alguien que estudie una carrera que no es su prioridad, no será un profesional feliz ni eficaz.

El sistema educativo debe crecer en los ámbitos organizativo, administrativo, presupuestario, académico, curricular, epistemológico, docente, estudiantil, material e infraestructural, para constituirse en un campo atractivo para los estudiantes, y que les permita egresarse del sistema estando, pertinentemente, preparados, tanto para obtener un mejor trabajo como para enfrentar y disfrutar la vida.

Como sistema establecido y dirigido por seres humanos, el educativo está y estará propenso a errores por diversos factores; pero lo problemático no son las equivocaciones, sino la poca efectividad para detectarlas y hacer, de manera expedita, los ajustes necesarios. Las dificultades que sí son divisadas, no se optimizan en corto tiempo, y, en el caso de las propuestas innovadoras, para mejorar o cambiar cierta condición deficiente, no son, por lo general, bien vistas, no son desarrolladas, de manera eficiente o, simplemente, se pierden en un largo proceso burocrático para su autorización. Esas innovaciones se ven enfrentadas con obstáculos de todo tipo, entre ellas: resistencias propias de los individuos, de las organizaciones educativas, del proceso instructivo en sí y de los sistemas educativo y social.

No es correcto pensar que un proceso de innovación no vaya a enfrentar resistencia de alguna índole; pero eso no implica que no se deba generar y ejecutar la innovación. Desde su planificación, el proceso de innovación debe estar confeccionado para las posibles resistencias que puedan presentarse. Se debe considerar que, en cuanto al fenómeno de la resistencia, “...para innovar es necesario conocerlo y, por tanto, diseñar estrategias destinadas a vencer las resistenciasobstáculos a la innovación" (Torre, 1998); asimismo, se debe pensar que los orígenes de ésta pueden ser variados.

Otro aspecto del sistema educativo, que lo hace propenso a errores, es que el establecimiento de algunas de las propuestas de mejoramiento o regulaciones educativas se presentan de forma impositiva, y provienen de personas, muchas veces, ajenas al ámbito educativo, las que por cuestiones políticas o económicas, plantean situaciones que complican el desarrollo de la enseñanza y del aprendizaje en las aulas, acotan la calidad de la educación, desestiman los valores claves para el crecimiento personal del niño y del joven, o descontextualizan la educación de la realidad.

Es necesario acudir a los grupos de personas que tienen relación con las realidades educativas, con los que están inmersos día a día en el quehacer educativo, cualesquiera que sea el nivel, desde el preescolar hasta el universitario, para construir propuestas más viables y oportunas a la realidad educativa que se vivencia. Se requiere de consenso y de trabajo en equipo entre los grupos de docentes y de estudiantes, quienes son los que se encuentran más involucrados en el proceso educativo, con las diferentes entidades relacionadas con la educación del país, desde las Universidades estatales y privadas, el Ministerio de Educación Pública (MEP), el Colegio de Licenciados y Profesores (COLYPRO) y otros colegios gremiales, hasta el Consejo Nacional de Rectores (CONARE), Unidad de Rectores de las Universidades Privadas en Costa Rica (UNIRE), Consejo Nacional de Educación Superior Privada (CONESUP), instituciones parauniversitarias estatales y privadas, por mencionar algunas de las entidades más relevantes. Esto con el fin de que los planteamientos o reformas, realmente, respondan a las necesidades del país y a las perspectivas de los niños y jóvenes. 
El Ministerio de Educación debe, entre otros aspectos, velar por mejorar y contextualizar los currículos; la contratación, la evaluación, la educación continua y la realimentación del personal docente; la infraestructura y los recursos tecnológicos y didácticos para los centros educativos; el nombramiento y la capacitación de las personas encargadas de los puestos administrativos, (asesores, supervisores, directores regionales, directores de escuelas y colegios) para que dirijan y organicen, eficientemente, el sistema educativo en los diferentes niveles, con humanismo, conocimiento y capacidad de liderazgo.

Otra situación importante, es el evitar conflictos por asuntos presupuestarios. Si bien, el gobierno ha logrado una relevante mejoría al aprobarse la reforma del artículo 78 de la Constitución Política para el Fortalecimiento de la Educación ${ }^{3}$, mediante la asignación de, al menos, un 8\% del Producto Interno Bruto (PIB) del presupuesto anual a la educación, con el fin de disponer de suficientes recursos para garantizar un acceso universal a la educación de calidad, puede suceder que esto no se cumpla fielmente o no se distribuya de manera eficaz.

Entonces, no debe discutirse sólo por lo mucho o lo poco que esto constituye, sino se debe supervisar su cumplimiento; pero lo primordial es optimizar los recursos con los que se cuenta actualmente o con los que se va a disponer en años siguientes, porque lo más importante es el recurso humano, y éste lo tenemos, tanto en la población estudiantil como en el personal docente. Para ello, reitero, también, la importancia de dirigir esfuerzos en el mejoramiento de la preparación de los docentes en todos los niveles, tanto en el sector público como en el privado, pues las certificaciones universitarias para laborar como docente son aceptadas por el Servicio Civil, aunque el nivel de formación no sea el mismo en los diversos recintos universitarios. Se destaca, entonces, de forma especial, la importancia de reforzar el apoyo a la educación superior pública dentro del presupuesto del $8 \%$ del PIB.

El enseñar y el aprender no es fácil, el organizar y mejorar el sistema educativo es dificultoso e implica un trabajo arduo, pero si se valoran propuestas viables, y no se estancan en planes de trabajo desproporcionados a la realidad costarricense, y que no han respondido a los requerimientos actuales, se pueden lograr mejoras significativas. Para conseguir adelantos, se debe profundizar en la preparación docente, en la efectividad del currículum, en la búsqueda de estrategias metodológicas más oportunas, en la descentralización de funciones, en la flexibilidad institucional para contar con su proyecto educativo de centro, en requisitos de salida diversos a los actuales para los diferentes ciclos o, al menos, en pruebas finales contextualizadas a la realidad de cada institución o región educativa, en analizar propuestas educativas de avanzada de otros países para adaptarlas a nuestra realidad, en investigar más sobre cómo aprende el ser humano y lograr explotar sus potencialidades. En fin, todo es un proceso que requiere análisis, propuestas renovadoras, sistematizaciones, valoraciones del papel de cada uno de los miembros de la comunidad educativa, mejoras en gestión administrativa, presupuestaria y curricular y alcances metodológicos progresivos, entre otros.

Es un reto para todos aquellos que estamos inmersos en el campo educativo, generar posibilidades de innovar, mejorar y reconstruir nuestro sistema educativo, desde cualquier ámbito y en cualquier nivel de cobertura, diagnosticando los fenómenos de la realidad educativa, generando procesos de investigación y presentando, desde nuestra posición laboral, propuestas de cambio en todos los niveles, que surjan de un trabajo conjunto de construcción y de toma de decisiones, y que se catalogue cada entidad educativa o departamento adscrito al Ministerio de Educación como una unidad fundamental de cambio.

En el Art. 78 de la Constitución Política se señala que en la educación estatal, incluida la superior, el gasto público no será inferior al ocho por ciento (8\%) anual del producto interno bruto, de acuerdo con la ley, sin perjuicio de lo establecido en los otros artículos 84 y 85 de esta constitución, según reforma de la Asamblea Legislativa de mayo de 2010. 
Toda propuesta, desde la formulada por una docente o un docente, hasta la proyectada por el Despacho del Ministro de Educación, debe ser valorada y considerada, pues puede servir como activador de otras y mejores propuestas de cambio. Lo importante es el deseo por mejorar y alcanzar un enriquecimiento auténtico e integral de nuestra educación, que genere siempre una perspectiva de cambio y de perfeccionamiento constante en nuestro desarrollo educativo.

\section{Referencias bibliográficas}

Costa Rica, Ministerio de Educación Pública. (2009). Reforma integral de las normas reguladoras de la promoción y repitencia dentro del sistema educativo público costarricense. Decreto n 34886-MEP. San José, Costa Rica: Despacho del Ministro de Educación, MEP.

Dengo, M. E. (1995). Educación costarricense. San José, Costa Rica: EUNED.

Gamboa, E. y Vásquez, G. (1998). Vamos a la secundaria. Estudios Sociales. México D. F.: McGraw-Hill.

Molina, I. y Palmer, S. (2007). Historia de Costa Rica. Breve, actualizada y con ilustraciones. San José, Costa Rica: Editorial Universidad de Costa Rica.

Programa Estado de la Nación en Desarrollo Humano Sostenible (Costa Rica). (2003). Décimo Informe Estado de la Nación en Desarrollo Humano Sostenible. Programa Estado de la Nación. San José, Costa Rica: El Programa.

Programa Estado de la Nación en Desarrollo Humano Sostenible (Costa Rica). (2007). Decimotercer Informe Estado de la Nación en Desarrollo Humano Sostenible. Programa Estado de la Nación. San José, Costa Rica: El Programa.

Programa Estado de la Nación en Desarrollo Humano Sostenible (Costa Rica). (2008). Decimocuarto Informe Estado de la Nación en Desarrollo Humano Sostenible. Programa Estado de la Nación. San José, Costa Rica: El Programa.

Programa Estado de la Nación en Desarrollo Humano Sostenible (Costa Rica). (2009). Decimoquinto Informe Estado de la Nación en Desarrollo Humano Sostenible. Programa Estado de la Nación. San José, Costa Rica: El Programa.

Salazar, J. (editor). (2003). Historia de la educación costarricense. San José, Costa Rica: EUNED.

Torre, S. (coordinador). (1998). Cómo innovar en los centros educativos. Estudio de casos. Madrid, España: Editorial Escuela Española. 